\title{
Representaciones Sociales de la Segunda Guerra Mundial: Valores y Dominancia social
}

\author{
Fernanda Mariel Sosa ${ }^{1}$, Dafna Gabriela Natapof ${ }^{2}$ y Elena Mercedes Zubieta ${ }^{3}$
}

\begin{abstract}
RESUMEN
Desde la perspectiva de las Representaciones Sociales (RS), surge una línea de investigación que considera la Historia como objeto de representación. Estudios previos muestran la Segunda Guerra Mundial (SGM) como un elemento nuclear de la historia universal. El objetivo del presente trabajo es describir la estructura representacional de la SGM, sus efectos, el perfil psicosocial en valores, creencias en la dominancia y contradominancia social y la disposición a luchar por el país. Por otro lado, se propone verificar si existen asociaciones entre los efectos de la SGM y las creencias, que tiene un grupo de población general $\left(n=224 ; 59.8 \%\right.$ mujeres, $40.2 \%$ hombres; $M_{\text {edad }}=30.5, D E=$ 10.68, Min $=18$, Máx $=65$ ). Los resultados muestran que la estructura de la RS de la SGM está organizada otorgándole centralidad a la muerte y a características de esta guerra en particular, como el nazismo, Hitler, los campos de concentración y exterminio, y las bombas atómicas. Los participantes consideran a la SGM como una catástrofe social, que además contribuyó a la formación de las Naciones Unidas y a la declaración de los derechos humanos. A su vez, priorizan valores de apertura al cambio y autotrascendencia, exhiben niveles altos de contradominancia social y una baja disposición a luchar por el país.

Palabras Clave: representaciones sociales, segunda guerra mundial, valores, dominancia social.
\end{abstract}

\section{Social Representations of the World War Two: Values and Social dominance}

\begin{abstract}
From the perspective of the Social Representations (SR), appears an investigation line that takes history as a representational object. Previous studies show World War II (WWII) as a central element of universal history. The purpose of this study is to describe the representational structure of the WWII, its effects, the psychosocial profile in values, beliefs in social dominance and counter-dominance and willingness to fight for the country. On the other hand, it is proposed to verify if there are associations between the effects of WWII and beliefs, which have a group of general population ( $n=224,59.8 \%$ women, $40.2 \%$ men, $M_{\text {age }}=30.5, S D=10.68$, Min = 18, Max =65). The results show that the structure of the SR of the WWII is organized giving centrality to death, and to characteristics of this particular war, such as Nazism, Hitler, concentration and extermination camps, and atomic bombs. Participants consider the WWII as a social catastrophe, which also contributed to the formation of the United Nations and the Declaration of Human Rights. In addition, they prioritize values of openness to change and self-trascendence, exhibit high levels of social counter-dominance and a low willingness to fight for the country.
\end{abstract}

Keywords: social representations, world war two, values, social dominance

\footnotetext{
1 y 3 Consejo Nacional de Investigaciones Cientificas y Técnicas (CONICET), Facultad de Psicologia Universidad de Buenos Aires, Argentina; fernandamarielsosa@hotmail.com

2 Facultad de Psicología Universidad de Buenos Aires, Argentina.
} 
Las Representaciones Sociales (RS; Moscovici, 1961) de la Historia y la Memoria Colectiva (MC; Halbwachs, 2004) explican la forma en que los grupos sociales perciben su pasado común, construyen su identidad social y edifican sus normas y valores en el presente, y se proyectan en el futuro (Liu \& Hilton, 2005).

Estudiar la construcción social de la memoria en relación con la Segunda Guerra Mundial (SGM) es relevante ya que es uno de los hechos históricos más recordados por los individuos de distintos países del mundo (Liu et al., 2005; Pennebaker, Paez \& Deschamps, 2006; Zubieta \& Barreiro, 2014), a su vez porque interpela identidades y permite un acercamiento a la comprensión de cómo el conocimiento actual de eventos sucedidos en el pasado pueden modificar actitudes, valores, creencias y conductas, de manera individual y social en el presente y el futuro (por ejemplo, relacionadas a la discriminación y la tolerancia, al prejuicio, a la coexistencia, entre otros).

Desde la perspectiva de la teoría de las RS surge una línea de investigación que considera a la historia como objeto representacional, e indaga cómo las personas y los grupos sociales se representan a sí mismos su pasado individual y social. En este marco, se hace relevante el concepto de $\mathrm{MC}$, el cual refiere al conjunto de representaciones del pasado que un determinado grupo produce, conserva, elabora y trasmite a través de la interacción de sus miembros (Valencia \& Páez, 1999). La MC es entonces considerada no sólo como la influencia de factores psicosociales en la memoria individual, sino también como aquello que da cuenta de la existencia de procesos psicológicos que superan la esfera individual e intersubjetiva. Refiere a la instancia interaccional en la que emerge el recuerdo de los grupos, naciones y etnias. La actividad de recordar constituye un contexto que influirá sobre los antecedentes y efectos de la memoria individual (Páez, Valencia, Pennebaker, Rimé \& Jodelet, 1998).

Las RS son definidas por Moscovici (1961) como una forma particular de conocimiento co-construido, que posibilita orientarse a través de la elaboración de comportamientos y la comunicación interpersonal, volviendo inteligible la realidad física y social, así como posibilita integrarse en grupos y en los intercambios habituales. Estas representaciones se estructuran mediante los procesos de interacción dentro del grupo social al cual los individuos pertenecen.

Existen distintas líneas de investigación de las diferentes escuelas dedicadas al estudio de las RS. Las principales son la Escuela Clásica, desarrollada principalmente por Moscovici y retomada por Jodelet, la Escuela de Ginebra, desarrollada por Doise, y la Escuela de Aix-en-Provence, desarrollada por Flament, y luego por Abric (Araya Umaña, 2002). Desde esta última línea, se estructurará el presente estudio.

Abric (2001) plantea, desde un enfoque estructuralista, que toda representación es una construcción socio-cognitiva integrada y organizada por un núcleo central y por elementos periféricos. El núcleo central implica uno o varios elementos que son los que dan el significado a la representación. Es lo más compartido por todos los miembros del grupo, está relacionado con el contexto global socio-histórico, y es lo más estable, por lo que es lo más resistente al cambio. Los elementos periféricos son los más flexibles, están más relacionados al contexto inmediato, y protegen la estabilidad de la representación. Poseen una jerarquía, es decir que cuánto más cerca están del núcleo central más se relacionan con el significado de la representación y, 
cuanto más lejos estén del mismo, aclaran y justifican esa significación. El sistema periférico permite la integración de la representación social con las variables de la experiencia individual.

\section{Representaciones Sociales de la Historia}

En los últimos años surgió una línea de investigación que considera la Historia como objeto representacional, indagando cómo las personas y los grupos sociales construyen su pasado, su presente y su futuro. Esto condiciona la forma en que definen su identidad, sus normas, sus valores, su relación con otros y su manera de afrontar los desafios que surgen (Liu \& Hilton, 2005).

Los grupos sociales cuentan con una memoria colectiva (Halbwachs, 2004) que implica una historia del pasado, un recuerdo y un olvido selectivo en función de la justificación del presente del grupo y de la predicción del futuro.

Estudios transculturales previos señalan la presencia de ciertos sesgos al recordar eventos de la historia universal. Entre ellos se encuentran: el sesgo bélico, que implica que los sucesos más mencionados están relacionados con guerras, revoluciones y politica; el sesgo eurocéntrico/ americano, que alude a la mayor evocación de eventos relacionados con la historia occidental; el sesgo nostálgico, es decir, recordar y calificar como positivos eventos lejanos en el tiempo; el sesgo de recencia, en el que se consideran como sucesos más importantes a los ocurridos en los últimos años; y el sesgo sociocéntrico, que refiere a otorgarle a eventos nacionales una relevancia a nivel mundial (Pennebaker et al., 2006; Techio et al., 2010).

El sesgo bélico es confirmado por la importancia que los participantes en diversos estudios (Liu et al., 2005; Pennebaker et al., 2006; Zubieta \& Barreiro, 2014) le adjudican a la SGM como primordial en la historia universal, junto a la figura de Hitler como el personaje más evocado y con la evaluación más negativa.

En cuanto a la consideración de los individuos respecto de la SGM, un estudio realizado en las naciones "victoriosas" como Estados Unidos y Rusia, da cuenta de la representación de la SGM como una Guerra Justa (Páez et. al., 2008). Mientras que otro estudio realizado en Argentina, un país posicionado como neutral la mayor parte de la guerra, muestra una representación que abarca desde el ser una catástrofe social (por las muertes y la destrucción) hasta la valoración de sucesos posteriores como la formación de la Organización de las Naciones Unidas y la Declaración de los Derechos Humanos, los avances científicos y tecnológicos, y la reconstrucción de la economía y las democracias (Zubieta \& Barreiro, 2014).

\section{Sistemas de valores y creencias}

Las RS en general, y las de la historia en particular, cuentan en su interior con sistemas de valores, de conocimientos y de prácticas que tienen la doble función de ordenar el mundo de modo que sea más accesible, guiar los actos en el entorno físico y social en el que una persona se desarrolla, así como de proponer un código compartido de significaciones que ayude a comunicarse, a nominar y clasificar sin ambigüedad los múltiples aspectos del mundo y de la historia (Farr, 1986). 
De acuerdo con Schwartz y Barnea (1995), los valores emergen como "metas deseables, transituacionales, variables en importancia, que sirven de principios rectores en la vida de la gente" (p. 17). Responden a necesidades individuales en tanto organismos biológicos, así como a requerimientos para la interacción social ordenada y el buen funcionamiento de los grupos. Son motivos sociales que se adquieren en la socialización, por lo que se vinculan a metas y fines deseables del grupo cultural de pertenencia. Los valores promueven, orientan e intensifican la acción relativamente estable por lo que se constituyen en normas de evaluación y justificación de las conductas. Es decir que las personas evalúan a otras personas, comportamientos, hechos, etc., según si estos están a favor o en contra de los objetivos que cada uno valora. Schwartz (2001) encuentra diez tipos motivacionales de valores que se organizan en dos dimensiones bipolares: apertura al cambio versus conservación, y autopromoción versus auto-trascendencia. Los valores de autopromoción hacen énfasis en el individuo mientras que los de autotrascendencia, en lo social.

La Apertura al cambio combina los valores de auto-dirección (pensamiento y acción independiente, con creatividad y exploración) y estimulación (entusiasmo, novedad y desafios en la vida). La Conservación incluye la seguridad (armonía, seguridad, y estabilidad de la sociedad, las relaciones y sí mismo), conformidad (restricción de las acciones, inclinaciones e impulsos que pudieran molestar o lastimar a otros y violaran las expectativas o normas sociales) y tradición (respeto, compromiso y aceptación por las costumbres e ideas que la religión o la cultura proporciona). La autotrascendencia implica el universalismo (entendimiento, aprecio, tolerancia y protección por el cuidado de todas las personas y la naturaleza) y la benevolencia (preservación e intensificación del cuidado de aquellos con quienes uno está en contacto personal frecuente). La auto-promoción combina el poder (estatus social y prestigio, control o dominancia sobre otras personas y recursos) y el logro (éxito personal a través de la demostración de competencias acordes a los criterios sociales). El hedonismo (placer y gratificación sensual para uno mismo) comparte elementos tanto del aperturismo como del interés propio.

La teoría explica la estructura de las relaciones dinámicas entre los valores. Es decir, da cuenta de cómo las acciones responden a la adhesión a un valor que puede ser congruente o entrar en conflicto con otros valores.

Junto a los valores hay también un conjunto de creencias que conciernen a las relaciones intergrupales. La teoria de la Dominancia Social (Social Dominance Orientation, SDO) postula que en todas las sociedades surge el conflicto entre grupos como una dimensión inevitable de la vida social. Para la reducción de este y de garantía de supervivencia, las sociedades y los grupos llegan a crear mitos de legitimación que sostienen la desigualdad, resaltando la superioridad de un grupo sobre otro, como es el caso del prejuicio étnico, el nacionalismo, el sexismo, la meritocracia y el conservadurismo económico-político (Pratto, Sidanius, Stallworth \& Malle, 1994). Existen dos variedades que condicionan los mitos de legitimación: (a) aumentar la jerarquía social, que implica justificar el incremento de la inequidad entre los grupos; y (b) atenuar la jerarquía social, que supone incrementar la igualdad entre los grupos sociales (Federico, 1999). De formulación reciente (Morselli et al., 2012), surge también la Orientación a la Contra-Dominancia (Counter-Dominance Orientation, CDO) como la 
contracara de la SDO, evaluando las respuestas psicológicas ante la opresión de los sistemas jerárquicos. La CDO está motivada por un deseo de inclusión y de pertenencia colectiva que, entre otros, indaga cómo las personas se movilizan contra la opresión y cómo se ganan adeptos para llevar a cabo dichas movilizaciones sociales.

Hallazgos de estudios transculturales previos permiten observar ciertos sesgos al recordar eventos de la historia, es decir, regularidades en la forma en que la gente recuerda la historia del mundo. Entre ellos se pueden encontrar el sesgo bélico, que implica que los eventos más mencionados están relacionados con guerras, revoluciones y política; el sesgo americano/eurocéntrico, donde son mencionados eventos relacionados con la historia occidental; el sesgo nostálgico, es decir, recordar y calificar como positivos eventos lejanos en el tiempo, y como negativos hechos cercanos; el sesgo de recencia, donde se observa como eventos importantes a los ocurridos en los últimos años (probablemente porque los han vivido ellos mismos, o se los contaron sus familiares que lo vivieron); y el sesgo sociocéntrico, considerando eventos nacionales como importantes universalmente (Pennebaker et al., 2006; Techio et al., 2010).

Estudios realizados a nivel local e internacional confirman el sesgo bélico como uno de los principales entre los recién nombrados (Liu et al., 2005; Pennebaker et al., 2006; Zubieta \& Barreiro, 2014), ya que al preguntar sobre los eventos más importantes de la historia, aparecen con mayor frecuencia eventos asociados a guerras y a la politica, a los que se vincula con la expresión "la violencia como partera de la historia". Parece haber por lo menos dos razones para esto (Liu \& László, 2007). La primera, es que es fácil relatar conflictos, donde se encuentran involucrados héroes y villanos, con un comienzo, un desarrollo y un final. La segunda, es que los conflictos generan emociones profundas, y se debería "recordar colectivamente aquellos sucesos nacionales o internacionales que hayan afectado de forma importante a sus vidas" (Pennebaker \& Basanick, 1998, p. 32).

La SGM -junto al holocausto y al nazismo- es el evento más mencionado al hablar de la historia universal, siendo Hitler el personaje más evocado, por lo que se puede observar que la SGM conforma un elemento nuclear de las RS de la historia, y podría considerarse una representación hegemónica de la historia mundial (Liu et al., 2005; Pennebaker et al., 2006; Zubieta \& Barreiro, 2014). En este sentido, el presente estudio se propone profundizar en el análisis del sesgo bélico en las RS en torno a la SGM, en términos de la estructura central y periférica en la que se organizan.

Para tal fin, se realizó un estudio empírico con el objetivo general de analizar la estructura representacional de la SGM. Como objetivos específicos se plantearon describir las RS de la SGM en función de su consideración y efectos, explorar el perfil psicosocial de los individuos en términos de valores y creencias en la dominancia y contra dominancia social y disposición a luchar por el país, y analizar la relación entre los efectos de la SGM y el perfil psicosocial en valores y creencias en la dominancia y contra dominancia social. 


\section{MÉTODO}

\section{Participantes}

Población general de ambos sexos, de entre 18 y 65 años, residentes en Argentina.

Tipo de estudio: exploratorio, descriptivo y de diferencias entre grupos.

Muestra: no probabilistica, intencional. Compuesta por 224 participantes, de las cuales el $59.8 \%$ son mujeres $(n=134)$ y el $40.2 \%$ son hombres $(n=90)$. La media de edad fue de $30.5(D E=10.68 ;$ Mín $=18$, Máx $=$ 65). El $62.1 \%(n=139)$ vive en Capital Federal y Conurbano bonaerense, mientras que el $37.9 \%(n=85)$ vive en el interior del país. El 63.8\% $(n=143)$ se autopercibe ideológicamente de izquierda, el 22.3\% $(n=50)$ de centro, y el $13.8 \%(n=31)$ de derecha. En cuanto a la religión, el $22.8 \%(n=51)$ se considera católico, el 25\% $(n=56)$ judio, el 25.9\% $(n=58)$ ateo, el $13.4 \%(n=$ $30)$ indiferente y el $12.9 \%(n=29)$ de otras religiones.

\section{Materiales}

Se utilizó un cuestionario auto-administrado aplicado de forma individual, anónima y voluntaria. Se indagó sobre datos sociodemográficos como edad, género y datos psicosociales como auto-posicionamiento ideológico y religión. Se les solicitó que mencionaran las primeras cinco ideas que se le venían a la mente cuando pensaban en la SGM (método de asociación libre). Además, se utilizó un instrumento del cual se seleccionaron para el presente trabajo las siguientes preguntas:

Dominancia y Contra Dominancia Social (Sidanius et al., 2004). Presenta frases donde se evalúa el grado de adhesión a un esquema referencial basado en la desigualdad entre los grupos que conforman la sociedad, mediante una escala Likert de 1 (totalmente en desacuerdo) a 7 (totalmente de acuerdo), con frases como "El valor que tienen algunos grupos de personas es mayor que el de otros", "La igualdad entre grupos de personas debería ser nuestro ideal". Los coeficientes de consistencia interna de la escala fueron: Dominancia social: alfa de Cronbach $=.85$ y Contradominancia Social: alfa de Cronbach $=$ .88

Valores (Schwartz, 2012). Versión reducida de 21 items. Recoge enunciados sobre valores o medios y fines deseables en la vida, que orientan la conducta social. El participante tiene que marcar la frase que mejor refleje cuánto se ve reconocido en cada enunciado -1 (no se parece nada a mî) a 7 (se parece mucho a $m \hat{\imath}$ )-. Luego los valores pueden agruparse en 10 tipos valóricos (benevolencia, universalismo, seguridad, tradición, conformidad, hedonismo, poder, logro, autodirección y conformismo) y estos en las cuatros dimensiones básicas que describe la teoría (autotrascendencia en oposición a la autopromoción y apertura al cambio en oposición a la conservación). Ejemplo de ítems: "Es muy importante para él/ella ayudar a la gente que lo rodea", "Se preocupa por su bienestar", "Anda siempre en busca de aventuras y le gusta arriesgarse", "Tener una vida llena de emociones es importante para él/ella". El coeficiente de consistencia interna de la escala fue: alfa de Cronbach $=.76 \mathrm{y}$ el de las subescalas: Universalismo: alfa de Cronbach $=.52$, Tradición: alfa de Cronbach $=.63$; Seguridad: alfa de Cronbach $=.55$; Hedonismo: alfa de Cronbach $=.63$; Estimulación: alfa de Cronbach $=.63$; Autodirección: alfa de 
Cronbach $=.41 ;$ Conformismo alfa de Cronbach $=.60 ;$ Benevolencia: alfa de Cronbach $=.55$; Logro: alfa de Cronbach $=.78$; Poder: alfa de Cronbach $=.57$.

Consideraciones y efectos de la SGM. Indaga sobre la importancia atribuida a los principales efectos de la guerra y el significado de cada uno, mediante una escala Likert de 1 (muy en desacuerdo) a 7 (muy de acuerdo). Ejemplo de ítems "Una guerra necesaria (fin de la colonización e inicio de la independencia para muchas naciones)", "Una guerra justa (para parar el nazismo, el fascismo y agresión japonesa)", "Una catástrofe social (muertos, destrucción, sufrimiento humano)". El coeficiente de consistencia interna fue alfa de Cronbach $=.73$

Disposición a luchar por su país. Se evalúa la disposición a luchar por su país/nación en el caso de que hubiera otra guerra, pidiéndole que elija la respuesta adecuada de 1 (definitivamente no) a 7 (por supuesto que sî).

\section{Procedimiento}

El cuestionario fue aplicado de manera online, en un periodo de 120 días aproximadamente. Los datos fueron recolectados a través de internet, contactando a conocidos, que a su vez contactaron a otros (método bola de nieve), proporcionándoles un link a partir del cual podían acceder al cuestionario. Antes de comenzar con las preguntas, se les indicaba que la participación era anónima y voluntaria, que la información sería utilizada solo con fines académicos, y que al comenzar la encuesta estaban dando su consentimiento informado.

Análisis de datos: para el análisis de la estructura de la representación, con el fin de reducir la dispersión de la información, aquellos elementos que referian a la misma idea fueron agrupados en la misma categoria, nombrando a esta a partir del término con mayor frecuencia de aparición. Por ejemplo, se utilizó el término "holocausto" para referir al mismo, como también a la evocación "shoá" (palabra en hebreo que designa a este suceso) o bajo "campos de concentración y exterminio" a las ideas de "Auschwitz" o "Treblinka". A partir de ello, se obtuvieron los datos de la estructura representacional con relación al núcleo central y periferia, mediante el software "Evoc" (Vergès, 1994) que permite ordenar los elementos en función de la frecuencia con que han sido mencionados y su orden de evocación. Para el análisis descriptivo y de correlación se utilizó el software SPSS.

\section{RESULTADOS}

Luego del proceso de reagrupación de elementos evocados, para el análisis de la información quedó un total de 114 evocaciones distintas, cuya distribución exhibe los siguientes puntos de corte: frecuencia mínima $=13 \mathrm{y}$ rango medio $=2.5$. Sobre estos criterios se construyen los cuadrantes en función de "rango por frecuencia" (ver tabla 1). 
Tabla 1

Estructura de la representación social de las ideas principales asociadas a la SGM en una muestra argentina.

\begin{tabular}{|c|c|c|c|c|c|c|}
\hline \multirow{9}{*}{$\begin{array}{l}\text { Rango } \\
\text { (Menor a 2.5) }\end{array}$} & \multicolumn{3}{|c|}{ Frecuencia (mayor a 29) } & \multicolumn{3}{|c|}{ Frecuencia (mayor a 13 y menor a 29) } \\
\hline & \multicolumn{3}{|c|}{ Núcleo Central } & \multicolumn{3}{|c|}{ Grupo Contraste } \\
\hline & \multirow{7}{*}{$\begin{array}{l}\text { Muerte } \\
\text { Nazismo } \\
\text { Hitler } \\
\text { Campos de } \\
\text { concentración y } \\
\text { exterminio } \\
\text { Bombas } \\
\text { atómicas }\end{array}$} & Frecuencia & Rango & & \multicolumn{2}{|c|}{ Frecuencia Rango } \\
\hline & & 72 & 2.125 & & 25 & 1.760 \\
\hline & & 33 & 2.061 & Injusticia & 24 & 2.333 \\
\hline & & 29 & 1.897 & \multirow{2}{*}{$\begin{array}{l}\text { Genocidio } \\
\text { Judíos }\end{array}$} & 22 & 1.591 \\
\hline & & & & & 19 & 2.263 \\
\hline & & 29 & 2.138 & $\begin{array}{l}\text { Intereses económicos } \\
\text { y políticos }\end{array}$ & 17 & 2.353 \\
\hline & & 29 & 2.448 & Racismo & 13 & 2.154 \\
\hline & \multicolumn{3}{|c|}{ Primera Periferia } & \multicolumn{3}{|c|}{ Segunda Periferia } \\
\hline & & Frecuencia & Rango & & Frecuen & Rango \\
\hline \multirow{5}{*}{$\begin{array}{l}\text { Rango } \\
\text { (Mayor a 2.5) }\end{array}$} & Destrucción & 41 & 2.610 & \multirow{2}{*}{$\begin{array}{l}\text { Poder } \\
\text { Tristeza }\end{array}$} & 23 & 2.826 \\
\hline & & & & & 23 & 2.957 \\
\hline & & & & Alemania & 19 & 2.632 \\
\hline & & & & Violencia & 16 & 2.750 \\
\hline & & & & Lucha & 13 & 2.692 \\
\hline
\end{tabular}

Los cuadrantes de la estructura circular del sistema de valores de Schwartz y Barnea (1995) refieren los distintos elementos de la estructura de la representación:

El núcleo central está conformado por los elementos con más frecuencia en su mención, y que aparecen en los primeros lugares al ser evocados. Este define la esencia de la representación. En este conjunto de elementos, se observa que la muerte es el término de mayor mención. La SGM terminó con la muerte de seis millones de judios, más de tres millones de prisioneros de guerra soviéticos, más de tres millones de católicos polacos, medio millón de gitanos, ochenta mil prisioneros politicos alemanes, setenta mil discapacitados mentales, más de diez mil homosexuales, y miles de testigos de Jehová (Rafecas, 2012). En segundo lugar aparece el Nazismo, un movimiento político y social cuyo nombre completo es "Partido Nacional Socialista Alemán de los Trabajadores". Este partido estaba liderado por Hitler, personaje mencionado como tercera evocación, quien asumió como canciller de Alemania en 1933, cargo que luego de la muerte del, en ese entonces, presidente Hindenburg, se unificara mediante una ley con el de presidente (Rafecas, 2012). El siguiente término mencionado es Campos de concentración y exterminio, a los que eran enviadas las distintas minorias (judíos, gitanos, testigos de Jehová, homosexuales, discapacitados mentales, presos politicos) con el objetivo de contar con mano de obra extremadamente barata y finalmente, eliminar a quienes "contaminaban la raza aria pura". En último lugar, aparecen evocadas las Bombas Atómicas. En agosto de 1945, armas nucleares cayeron sobre dos ciudades japonesas, Hiroshima y Nagasaki, provenientes de Estados Unidos, como respuesta al ataque recibido en Pearl Harbor (Hobsbawm, 2012).

La Primera Periferia está representada por los elementos con alta frecuencia de mención, pero nombrados en los últimos lugares. En este caso aparece una única evocación, la destrucción, y refleja que los años de guerra implicaron una gran cantidad de muertes, junto con la destrucción material 
de edificios y ciudades, y la aniquilación simbólica y cultural (quema de libros, objetos religiosos, etc.; Rafecas, 2012).

El Grupo Contraste alude a los elementos con baja frecuencia en su evocación, pero que figuran en los primeros lugares. Son elementos que podrían formar parte del núcleo central en un futuro. Se observa aquí con mayor frecuencia al Holocausto. Este es un término utilizado para mencionar la persecución y el exterminio de los judíos, dentro del cual se encuentran hechos como las leyes de Nuremberg en 1935, la Noche de los Cristales Rotos en 1938, la propaganda y la educación antijudia (como el libro para niños El hongo venenoso), el proceso de arización, de guetoización, y los campos de concentración, trabajo y exterminio (Rafecas, 2012; Steinfeldt, 2009). Le sigue la evocación de Injusticia, valor percibido en razón de toda la destrucción ocasionada por este conflicto bélico. En tercer lugar aparece Genocidio, que implica diversos actos realizados con la intención de destruir, total o parcialmente, a un grupo nacional, étnico, racial o religioso (Organización de las Naciones Unidas, 1948), coherente ya que en la Alemania nazi se intentó destruir sistemáticamente a diversos grupos sociales. En cuarto lugar están los Judíos, una de las principales minorias afectadas por el régimen nazi, seguido por los Intereses económicos y políticos que perseguía el nazismo, que ocupan el quinto lugar, y finalmente el Racismo, la visión de Hitler de que la "raza aria" era superior a las demás.

La Segunda Periferia se conforma por los elementos menos mencionados, que aparecen en los últimos lugares, y son más dependientes del contexto social inmediato. En primer lugar se observa el término Poder, el que era ostentado a gran escala por los dirigentes y militares del partido nazi. Luego aparece la Tristeza, sentimiento asociado a ideas como la muerte, la destrucción y la injusticia ocurridas en esta guerra. Le sigue la evocación Alemania, quien dio comienzo oficial a este conflicto al invadir Polonia en 1939. En los últimos lugares se encuentran los términos Violencia y Lucha, aludiendo probablemente por un lado a las ejercidas por los soldados y militantes nazis, y por otro, a la resistencia por parte de las minorias. Es destacable el rol de los movimientos juveniles en la resistencia cultural (educación, periódicos clandestinos) y armada (levantamiento del Gueto de Varsovia, entre otros) (Sinay, 2014).

Al continuar con el objetivo de describir las RS de la SGM en función de su consideración y efectos, se observa que los participantes priorizan la concepción de la SGM como una catástrofe social (que implica muertos, destrucción y sufrimiento humano), seguida de la mención de efectos como la Formación de las Naciones Unidas y la Declaración de los Derechos Humanos (ver tabla 2).

Tabla 2

Puntuaciones medias en las consideraciones y efectos de la SGM.

\begin{tabular}{|c|c|c|}
\hline Consideraciones y efectos SGM & M & $D E$ \\
\hline Una catástrofe social (muertos, destrucción, sufrimiento humano) & 6.54 & 1.21 \\
\hline Formación de las Naciones Unidas y Declaración de los Derechos del Hombre & 5.08 & 1.83 \\
\hline Guerra Fría (formación de los bloques comunista y capitalista) & 4.56 & 2.17 \\
\hline Avances científicos y tecnológicos & 4.13 & 2.04 \\
\hline Reconstrucción de la economía y de las democracias & 3.75 & 2.02 \\
\hline Una guerra justa (para parar el nazismo, el fascismo y agresión japonesa) & 2.31 & 1.80 \\
\hline Una guerra necesaria (fin de la colonización e inicio de la independencia para muchas naciones) & 2.11 & 1.60 \\
\hline
\end{tabular}


Con el propósito de explorar el perfil psicosocial de los participantes en términos de valores y creencias en la dominancia y contra dominancia social, y la disposición a luchar por el país, se realizó un análisis descriptivo que arrojó los siguientes resultados (ver tabla 3).

Tabla 3

Puntuaciones medias en SDO, CDO, valores y disposición a luchar por el país.

\begin{tabular}{lcc}
\hline & $\boldsymbol{M}$ & $\boldsymbol{D E}$ \\
\cline { 2 - 3 } Dominancia y Contradominancia Social & & \\
$\quad$ Contradominancia Social & 10.9 & 2.78 \\
$\quad$ Dominancia Social & $4, .62$ & 2.53 \\
Dimensiones subyacentes de valores & & \\
$\quad$ Apertura al cambio & 14.96 & 3.36 \\
$\quad$ Autopromoción & 11.61 & 3.76 \\
$\quad$ Conservación & 12.13 & 4.33 \\
$\quad$ Autotrascendencia & 16.18 & 2.64 \\
Disposición a luchar por su país/nación & 2.48 & 1.85 \\
\hline
\end{tabular}

Como se observa en la tabla 3, los participantes exhiben puntuaciones más elevadas en la creencia en la contra-dominancia social en comparación con la creencia en la dominancia, es decir, adhieren a la idea de la igualdad entre grupos. En relación con las dimensiones subyacentes de valores, sobresalen la auto-trascendencia y la apertura al cambio, delineando un perfil que da cuenta de que los participantes consideran importante ayudar a quienes los rodean, tener en cuenta sus necesidades, ser leales a los amigos, perdonar y no guardar rencor. Hay una orientación hacia el apoyo de la igualdad de oportunidades en la vida, a escuchar a quienes son distintos, proteger la naturaleza y adaptarse a ella sin alterarla, promover la justicia, la paz y la protección de los más débiles. A su vez, enfatizan la independencia de juicio y de acción, favoreciendo el cambio. Por último, y de manera coherente con lo hasta aquí descripto, se observa en los participantes puntuaciones bajas respecto de la disposición a luchar por su país/nación en el caso que ocurriera una guerra.

Posteriormente, se propuso verificar si existen correlaciones entre la dominancia y contra dominancia social, los valores y la disposición a luchar por el país/nación con las consideraciones y efectos de la SGM. Para tal fin se realizó una prueba $r$ de Pearson. Se encontraron asociaciones positivas entre el efecto de la SGM de favorecer avances tecnológicos, así como la formación de las Naciones Unidas y la Declaración de los Derechos del Hombre, con la creencia en la contra-dominancia social (ver tabla 4).

A su vez, quienes consideran que la SGM fue una guerra necesaria priorizan valores de apertura al cambio, conservación y auto-trascendencia. La consideración de que fue una guerra justa se asocia con valores de autopromoción y de conservación. El considerar a la SGM como una catástrofe social correlaciona de manera positiva con valores de auto-trascendencia. La postura de que la SGM favoreció los avances tecnológicos y científicos se asocia de manera positiva con valores de autopromoción. La consecuencia de la formación de las Naciones Unidas y la Declaración de los Derechos del Hombre se asocia con valores de autopromoción y conservación. Por último, pensar la reconstrucción de la economía y las democracias como 
consecuencias de la SGM se relaciona con valores de conservación (ver tabla $5)$.

Tabla 4

Correlaciones entre las consideraciones y efectos de la SGM y la dominancia y contradominancia social.

\begin{tabular}{lcc}
\hline Consideraciones y efectos SGM & CDO & SDO \\
\hline Guerra necesaria & .037 & .036 \\
Guerra justa & .017 & .033 \\
Catástrofe social & .109 & -.064 \\
Guerra Fría & .115 & -.104 \\
Avances científicos y tecnológicos & $.208^{* *}$ & .035 \\
Formación ONU y Declaración de los Derechos del Hombre & $.244^{* *}$ & -.099 \\
Reconstrucción de la economía y de las democracias & .032 & .050 \\
\hline
\end{tabular}

${ }^{* *} p<.010$

$* p<.050$

Tabla 5

Correlaciones entre las consideraciones y efectos de la SGM y las dimensiones subyacentes de valores.

\begin{tabular}{|c|c|c|c|c|}
\hline Consideraciones y efectos SGM & $\begin{array}{l}\text { Apertura } \\
\text { al cambio }\end{array}$ & $\begin{array}{l}\text { Auto } \\
\text { promoción }\end{array}$ & Conservación & $\begin{array}{l}\text { Auto } \\
\text { trascendencia }\end{array}$ \\
\hline Guerra necesaria & $.133^{*}$ & .130 & $.148^{*}$ & $.151^{*}$ \\
\hline Guerra justa & .008 & $.133^{*}$ & $.166^{*}$ & .112 \\
\hline Catástrofe social & .114 & -.006 & .020 & $.176^{\star *}$ \\
\hline Guerra Fría & .011 & .045 & -.128 & -.003 \\
\hline Avances científicos y tecnológicos & .116 & $.179^{* *}$ & .073 & .101 \\
\hline $\begin{array}{l}\text { Formación ONU y Declaración de los } \\
\text { Derechos del Hombre }\end{array}$ & .029 & $.222^{* *}$ & $.146^{*}$ & .077 \\
\hline $\begin{array}{l}\text { Reconstrucción de la economía y de las } \\
\text { democracias }\end{array}$ & .021 & .114 & $.142^{*}$ & .119 \\
\hline
\end{tabular}

** $p<.010$

$* p<.050$

Finalmente, se encontró que la disposición a luchar por el país en el caso de que ocurriese un conflicto bélico se asocia a concebir a la SGM como una guerra justa y necesaria (ver tabla 6).

Tabla 6

Correlaciones entre las consideraciones y efectos de la SGM y la disposición a luchar por el país (WTF).

\begin{tabular}{ll}
\hline Consideraciones y efectos SGM & WTF \\
\hline Guerra necesaria & $.150^{*}$ \\
Guerra justa & $.137^{*}$ \\
Catástrofe social & .004 \\
Guerra Fría & .034 \\
Avances científicos y tecnológicos & .106 \\
Formación ONU y Declaración de los Derechos del Hombre & .037 \\
Reconstrucción de la economía y de las democracias & .049 \\
\hline$* p<.010$ & \\
$* p<.050$ &
\end{tabular}

\section{DISCUSIÓN}

Diversas investigaciones muestran referencias a guerras, batallas y conflictos como sucesos centrales en la historia, lo que es denominado como 
sesgo bélico (Liu et al., 2005). Los hallazgos de este estudio contribuyen al estudio de las RS de la historia y, más específicamente, a profundizar en el análisis de uno de los eventos más nombrados en diferentes estudios transculturales (Liu et al., 2005; Pennebaker et al., 2006; Zubieta \& Barreiro, 2014), la Segunda Guerra Mundial, dentro de lo que puede considerarse como una representación hegemónica de la historia universal.

Las RS están organizadas en una estructura alrededor de un núcleo central y elementos periféricos. El núcleo central es el que da el significado a la representación, es lo más compartido por todos los miembros del grupo. Los resultados obtenidos muestran que la estructura de la RS de la SGM de un grupo de individuos argentinos está centrada en la muerte, inevitable en un conflicto de esta envergadura, junto a particularidades de este hecho como el nazismo, Hitler, los campos de concentración y exterminio y las bombas atómicas. Los elementos periféricos son los que permiten las variaciones individuales y que están ligados al contexto más inmediato. En la periferia de la RS de la SGM se encuentran ideas intimamente relacionadas a las del núcleo: la destrucción, el racismo, el genocidio, el holocausto, los judíos, la violencia y la lucha, se combinan con evocaciones a Hitler y el nazismo y su puesta en práctica en los campos de concentración y exterminio, sumado a su búsqueda por el poder como medio para dar respuesta a los intereses económicos y políticos. Todo enmarcado en sentimientos como la tristeza y valores como la injusticia.

Con respecto a la consideración de la SGM, y teniendo en cuenta que Argentina fue neutral la mayor parte de la guerra, los participantes la perciben mayoritariamente como una catástrofe social por las muertes, la destrucción y la violencia que conllevó, pero a su vez también señalan el que haya favorecido a la formación de las Naciones Unidas y la Declaración de los Derechos Humanos. Resultados similares se encontraron en un estudio previo realizado en Argentina en el que los participantes valoran a la SGM como una catástrofe social, sumado a la creación de la ONU, los avances científicos y tecnológicos, y la reconstrucción de la economía y las democracias (Zubieta \& Barreiro, 2014).

Teniendo en cuenta el perfil psicosocial, los participantes exhiben un perfil de valores en el que se prioriza la apertura al cambio y la autotrascendencia, es decir enfatizan la independencia de juicio y la acción, y la preocupación y aceptación de los otros como iguales. A su vez, y en coherencia con los valores, adhieren a la creencia en la igualdad entre los grupos, acuerdan con la inclusión y la pertenencia colectiva y se oponen a la opresión de los sistemas jerárquicos. Finalmente, muestran una baja disposición a luchar por su país en el caso de un conflicto bélico.

La posición respecto de la consideración de la SGM, en términos de que fue una guerra que favoreció los avances tecnológicos y la formación de la Organización de las Naciones Unidas y de la Declaración de los Derechos Humanos, se asocia con la creencia en la igualdad de los diversos grupos, en su coexistencia pacífica en la sociedad.

La creencia de que la SGM fue una guerra necesaria (es decir, que implicó el fin de la colonización e inicio de la independencia para muchas naciones) y una guerra justa (que sirvió para parar al nazismo, el fascismo y la agresión japonesa), se relaciona con la disposición a combatir por el país en caso de ser necesario. 
Los valores direccionan los objetivos que las personas estiman para sus vidas y para la sociedad y, por lo tanto, cómo evalúan lo que ocurre a su alrededor (Schwartz, 2012). Los participantes que priorizan valores relacionados a la apertura al cambio, es decir, que valoran la independencia de pensamiento y de acción, que buscan desafios constantemente y que muestran gusto por lo nuevo, consideran que la SGM fue principalmente una guerra necesaria para terminar con la colonización y dar lugar a la independencia de diversos países. A su vez, también la conciben así los participantes que valoran la conservación -el vivir en una sociedad estable, en armonía y seguridad, aceptando las ideas propuestas por la misma- y la autotrascendencia, el proteger, apreciar y tolerar a la naturaleza y a todas las personas. La preferencia por estos últimos valores se asocia también a la idea de considerar a la SGM como una catástrofe social, por el poco respeto que se tuvo en los años de guerra por las vidas humanas, la cultura y la sociedad en general. La prioridad que se otorga a valores de conservación, además, se asocia con resaltar la importancia de la reconstrucción de la economía y las democracias, la formación de la ONU y la Declaración de los Derechos Humanos, y a la creencia de que este conflicto bélico fue justo porque sirvió para parar los regímenes totalitarios de la época. Estas últimas consecuencias, junto con los avances tecnológicos y científicos que ocasionó, son priorizadas por los participantes que estiman la autopromoción, es decir, el éxito personal y el poder.

Las limitaciones del estudio se relacionan con la intencionalidad de la muestra, que no permite hacer extensibles los hallazgos a la población argentina en su totalidad. Sin embargo, se trata de un estudio exploratorio en una muestra argentina. Sería importante que futuros estudios profundicen el análisis, incorporando muestras de diferentes regiones del país. Otra limitación se encuentra en haber realizado el cuestionario vía online, ya que no permite dar cuenta de la responsabilidad del sujeto al responder.

Los resultados de esta investigación permiten profundizar el análisis del sesgo bélico en las RS de la historia. Este trabajo también contribuye al conocimiento de las RS y la MC en relación con uno de los eventos que podría considerarse una representación hegemónica de la historia universal, la SGM. Este recuerdo es una construcción basada en el presente del grupo social, que permite, a la vez, comprender hechos, normas y valores actuales, y predecir futuros. Por otra parte, el equipo de investigación del cual se desprende este trabajo se encuentra actualmente profundizando en las RS del sesgo bélico representado por otros elementos nucleares de la historia: la Primera Guerra Mundial (Sosa, Natapof \& Zubieta, 2017) y la Guerra de Malvinas.

\section{REFERENCIAS}

Abric, J. (2001). Prácticas sociales y representaciones. México: Ediciones Covoacén.

Araya Umañan, S. (2002). Las representaciones sociales: Ejes teóricos para su discusión. San José: Facultad Latinoamericana de Ciencias Sociales (FLACSO).

Farr, R. (1986). Las Representaciones Sociales. En S. Moscovici (Ed.), Psicología social (II): Pensamiento y vida social (pp. 495-506). Barcelona: Paidós.

Federico, C. (1999). The Interactive Effects of Social Dominance Orientation, Group Status, and Perceived Stability on Favoritism for High-Status Groups. Group Processes Intergroup Relations, 2(2), $119-143$. https://doi.org/10.1177/1368430299022002

Halbwachs, M. (2004). La memoria colectiva. Zaragoza: Prensas Universitarias de Zaragoza.

Hernández Sampieri, R., Fernández Collado, C. \& Baptista Lucio, P. (2003). Metodología de la investigación ( $3^{\circ}$ Edición). México: McGraw-Hill.

Hobsbawm, E. (2012). Historia del Siglo XX. Buenos Aires: Critica.

Liu, J. H., Goldstein-Hawes, R., Hilton, D., Huang, L., Gastardo-Conaco, C., Dresler-Hawke, E.,... Hidaka, 
Y. (2005). Social representations of events and people in world history across twelve cultures. Journal of Cross Cultural Psychology, 36, 171-191. https://doi.org/10.1177/0022022104272900

Liu, J. H. \& Hilton, D. (2005). How the past weighs on the present: Social representations of history and their role in identity politics. British Journal of Social Psychology, 44, 1-21. https://doi.org/10.1348/014466605X27162

Liu, J. \& László, J. (2007). A narrative theory of history and identity: Social identity, social representations, society and the individual. En G. Maloney \& I. Walker (Eds.), Social representations and identity: Content, process and power (pp. 85-107). London: Palgrave Macmillan.

Morselli, D., Pratto, F., Bou Zeineddine, F., Aranda, M., Lee Stewart, A., Cidam, A.,... Sweetman, J. (julio, 2012). Social Dominance and Counter Dominance Orientation Scales (SDO/CDO): Testing Measurement Invariance. The 35th Annual Meetings of the International Society of Political Psychology, Chicago.

Moscovici, S. (1961). El psicoanálisis, su imagen y su público. Buenos Aires: Huemul.

Organización de las Naciones Unidas (1948). Prevención y sanción del delito de genocidio. Recuperado de http://www.un.org/es/comun/docs/?symbol=A/RES/260(III)

Páez, D., Liu, J. H., Techio, E., Slawuta, P., Zlobina, A., \& Cabecinhas, R. (2008). Remembering World War II and willingness to fight: Socio-cultural factors in the social representation of historical warfare across 22 societies. Journal of Cross-Cultural Psychology, 39, 373-380. https://doi.org/10.1177/0022022108316638

Páez, D., Valencia, J. F., Pennebaker, J. W., Rimé, B., \& Jodelet, D. (1998). Memorias colectivas de procesos culturales y politicos. Bilbao: Servicio editorial de la UPV.

Pennebaker, J. \& Basanick, B. (1998). Creación y mantenimiento de las memorias colectivas. En D. Páez (Ed.), Memorias colectivas de procesos culturales y politicos. Bilbao: Universidad del País Vasco.

Pennebaker, J. W., Páez, D., \& Deschamps, J. C. (2006). The social psychology of history. Psicología politica, $32,15-32$.

Pratto, F., Sidanius, J., Stallworth, L. M., \& Malle, B. (1994). Social Dominance Orientation: A Personality Variable Predicting Social and Political Attitudes. Journal of Personality and Social Psychology, 67(4), 741-763. https://doi.org/10.1037/0022-3514.67.4.741

Rafecas, D. (2012). Historia de la solución final. Una indagación de las etapas que llevaron al exterminio de los judios europeos. Buenos Aires: Siglo Veintiuno Editores.

Schwartz, S. (2001). ¿Existen aspectos universales en la estructura de los valores humanos? En M. Ros, V. Gouveia. Psicología Social de los Valores Humanos. Desarrollos teóricos, metodológicos y aplicados. Madrid: Biblioteca Nueva.

Schwartz, S. (2012). An overview of the Schwartz Theory of Basic Values. Online Readings in Psychology and Culture, Unit 2(1). https:/ / doi.org/10.9707/2307-0919.1116

Schwartz, S. H. \& Barnea, M. (1995). Los valores en orientaciones politicas: Aplicaciones en España, Venezuela y México. Psicología Politica, 11, 15-40.

Sosa, F., Natapof, D., \& Zubieta, E. (2017). Representaciones sociales de la primera guerra mundial en estudiantes de psicologia e historia social. Anuario de investigaciones, 23, 173-180.

Sidanius, J., Pratto, F., van Laar, C., \& Levin, S. (2004). Social Dominance Theory: Its Agenda and Method. Political Psychology, 25(6), 845-880. https://doi.org/10.1111/j.1467-9221.2004.00401.x

Sinay, M. (2014). Resplandor en las tinieblas nazis. Retratos de la resistencia judia olvidada durante el Holocausto. Villa Maria: Editorial Universitaria Villa María.

Steinfeldt, I. (2009). ¿Cómo fue humanamente posible? Un estudio de perpetradores y espectadores durante el Holocausto. Jerusalén: Yad Vashem. La escuela internacional para el estudio del holocausto.

Techio, E., Bobowik, M., Páez, D., Cabecinhas, R., Liu, J., Zubieta, E. \& Espinosa, A. (2010). Social representations of history, wars and politics in Latin America, Europe and Africa. Revista de psicologia social, 25(1), 11-26. https://doi.org/10.1174/021347410790193441

Valencia, J. \& Páez, D. (1999). Generación, polémica pública, clima social y recuerdo de hechos políticos. Psicología Política, 18, 11-30.

Vergès P. (1994) Appoche du noyau central: proprietès quantitatives et structurales. En C. Guimelli (Ed.). Textes de Base in Sciences Sociales: Etructures et Transformation des Représentations Sociales (pp. 233253). Paris (PA): Delachaux et Niestlé.

Zubieta, E. \& Barreiro, A. (2014). Memoria Colectiva y Representaciones Sociales de la Historia. Un estudio preliminar con estudiantes universitarios argentinos. En E. Zubieta, J. F. Valencia \& G. I. Delfino (Eds.), Psicología social y politica: procesos teóricos y estudios aplicados. Buenos Aires: EUDEBA.

Recibido 01-07-2017 | Aceptado 10-08-2017

Este trabajo se encuentra bajo una Licencia Creative Commons Atribución 4.0 Internacional que permite a terceros utilizar lo publicado siempre que se dé el crédito pertinente a los autores y a Psicodebate 